\title{
Porosity prediction from offshore seismic data of F3 Block, the Netherlands using multi-layer feed-forward neural network
}

\author{
Prabodh Kumar Kushwaha ${ }^{1, *}$, S. P. Maurya ${ }^{2}$, Piyush Rai ${ }^{1}$ and N. P. Singh ${ }^{2}$ \\ ${ }^{1}$ Department of Mining Engineering, Indian Institute of Technology (BHU), and \\ ${ }^{2}$ Department of Geophysics, Institute of Science, Banaras Hindu University, Varanasi 221005 , India
}

In the present study, seismic and well log information is incorporated with a multi-layer feed-forward neural network (MLFN) to predict porosity in the inter-well region. The aim of this study is to estimate a relationship between porosity and impedance to characterize the reservoir, if any, in the offshore F3 block, the Netherlands. MLFN is used to generate a connection between porosity logs and a set of seismic attributes, which are further used for porosity prediction. Modelbased inversion is employed to produce an acoustic impedance volume, which is a reliable technique for quantitative estimation of reservoir characteristics and acoustic impedance. The model-based inversion results indicate that the acoustic impedance (AI) in the region varies from 2500 to $6200 \mathrm{~m} / \mathrm{s}^{*} \mathrm{~g} / \mathrm{cm}^{3}$, which is comparatively low and indicates loose formation. Thereafter, AI along with other attributes estimated from seismic data, is used as an input in MLFN, and porosity is predicted. The technique is first implemented on the traces close to well locations, and the findings are correlated with well log information, and after appropriate matching, the entire seismic segment is inverted for porosity. The results indicate that the porosity varies from 0.07 to 0.40 . Further, a relationship between predicted porosity and inverted impedance is derived to represent the connection between these two parameters in the region. Moreover, based on this study, it is concluded that there is no significant reservoir in the region. However, as the analyses are performed for a specific range of data, it is possible that other parts of the area may have a different stratigraphy and possibility of the primary reservoir in the area.

Keywords: Acoustic impedance, multi-layer feed-forward neural network reservoir, porosity, seismic inversion.

MAPPING of the physical features of the subsurface is essential for proper development of hydrocarbon reservoirs. Using well-logging tools or core samples, these characteristics, such as porosity, $P$-wave velocity, permeability, $S$-wave velocity, density, etc. can be evaluated at the well locations. However, the geological model developed

\footnotetext{
*For correspondence. (e-mail: prabodhkk.rs.min15@itbhu.ac.in)
}

using 3D interpolation of these measurements often does not satisfy the need of the development team. The reasons may be the sparse location of wells or the complexity of geology. In these circumstances, the $3 \mathrm{D}$ seismic survey offers the development region with more comprehensive coverage and option ${ }^{1}$.

The first step towards a successful hydrocarbon discovery is the reliable interpretation of seismic data and generation of high-resolution subsurface images ${ }^{2}$. The reflected seismic data provide the property of the interface and hence cannot be appropriately described in terms of subsurface lithology. Seismic data are band-limited in frequency, and seismic inversion provides only the relative (band-limited) impedance value ${ }^{3}$. Therefore, seismic inversion is used to measure acoustic impedance of the subsurface, which provides the properties of the layers and helps in the interpretation of the seismic section. Furthermore, as this acoustic impedance is not sufficient to provide adequate data for the characterization of the reservoir, other parameters such as porosity, permeability, shale volume, etc. are needed for this purpose ${ }^{4}$. These parameters can be predicted by the integration of seismic and well-log information using geostatistical techniques.

Geostatistical techniques are routinely used for the prediction of different geophysical parameters from seismic and $\log$ information and make use of two types of attributes, namely internal attributes estimated by the transformation of complex traces and external attributes derived from seismic inversion methods ${ }^{5-8}$. These techniques utilize sample points collected at various places and incorporate them into the seismic segment, where no $\log$ information is provided. These sample points are the petrophysical parameters measured in the boreholes ${ }^{8}$. There are two classes of geostatistical interpolation techniques, namely deterministic and geostatistical ${ }^{9}$. The deterministic technology of interpolation makes use of mathematical functions, while the geostatistical interpolation technique makes use of both statistical and mathematical methods ${ }^{10-12}$. The first use of geostatistical techniques for reservoir characterization, though useful, was restricted due to reliance on the well-log density requirement ${ }^{10}$. The recent techniques, nevertheless, implement an effective integration of high-frequency 
well-log data and low-frequency seismic data, and the inputs to geostatistical techniques no longer comprise conventional seismic reflection data and log data ${ }^{13,14}$.

Although there are many geostatistical methods available in the literature, in the present study we have used a multi-layer feed-forward neural network (MLFN) for computing the petrophysical parameters ${ }^{15,16}$ (porosity) because MLFN is fast and requires lesser space for storage of data. The study aims to establish an association between the attributes and porosity, and to characterize the reservoir in the area, if any. In the initial step, the MLFN method is implemented on a composite trace (the average of the neighbouring traces centred at the well location) near a well position to predict the porosity, and the result is validated by information from the well in that region ${ }^{10,17}$ In the next step, the whole seismic segment is converted into the porosity section employing the MLFN method.

The objective of the study is to estimate the porosity section of the area to characterize the reservoir, if any, and to establish a relationship between porosity and impedance. The objective is achieved by generating seismic attributes with the well-log data integration, termed as internal attributes, and impedance from seismic attributes termed as external attributes. Next, at the sample points, a relationship between attributes and porosity of well log is generated using seismic attributes and log porosity, which is further used for porosity prediction in the inter-well region ${ }^{16}$. The inversion is performed using Hampson Russell Software (HRS 10.2), and analysis is done in Matlab programming language.

\section{Study area}

The study area is located in the F3 block, North Sea, the Netherlands. The 3D offshore seismic data and well-log data have been used for the analysis. The F3 block coordinates are $\mathrm{N} 54^{\circ} 52^{\prime} 0.86^{\prime \prime} \mathrm{N} / 4^{\circ} 48^{\prime} 47.07^{\prime \prime} \mathrm{E}$. The survey was conducted in 1987, covering an area of $384 \mathrm{sq} . \mathrm{km}$. The F3 block is mainly situated on the top of the Dutch central graben (shelf of the North Sea) as one of the key Kimmerian rift basins in the Dutch northern offshore and a part of the structural component of the Mesozoic southern North Sea Rift System ${ }^{18}$. It is basically characterized by great horizontal and vertical variability. A threegenerational fault system is notable in the F3 block $^{19}$. The first generation has known to be oblique-slip, reversing, sinistral faults with SSW-NNE orientation. The second generation has oblique-slip, normal, dextral faults with $\mathrm{W}-\mathrm{E}$ orientation, and the third generation has faults disturbed by Permian halokinesis, which are genetically connected with the first- and second-generation faults. There are four vertical wells available in the area with sonic and gamma-ray logs, but the density log is available only for two wells, namely F02-1 and F03-2, with depth range approximately $1700 \mathrm{~ms}$ (ref. 15). The well loca- tions of F02-1 is 362 inline and 336 cross line, well F032 is 722 inline and 848 cross line, for well F03-4 is 442 inline and 1007 cross line and for well F06-1 is 244 inline and 387 cross line in the seismic section. The exposure of seismic survey ranges from inline 100 to 750 and cross line 300 to 1200 with a regular unit step. The size of the survey area is $24 \mathrm{~km}$ and $16 \mathrm{~km}$ in inline direction and cross line direction respectively, with a $25 \mathrm{~m} \times 25 \mathrm{~m}$ bin size. The two-way travel time record of seismic data is $1.848 \mathrm{~ms}$, with a sampling rate of $4 \mathrm{~ms}$. The primary F3 block dataset was noisy, and a dip-steered median filter with a two traces limit was used to reduce the noise. Figure $1 a$ represents the location map of the study area, along with the survey region and location of wells. Figure $1 b$ shows the stratigraphy of the North Sea region ${ }^{20}$.

In the Upper-Jurassic-Lower Cretaceous strata, the 3D seismic signal was acquired with the objective of gas and oil exploration. The dataset post $1200 \mathrm{~ms}$ comprises Miocene, Pliocene and Pleistocene reflectors ${ }^{21,22}$. The great sigmoidal bedding comprises deposits of a bulky structure of fluviodelta draining significant sections of the Baltic Sea $\operatorname{area}^{23,24}$. The deltaic bundle comprises of a giant sand and shale porosity $(20-40 \%)$ with some cemented marks of carbonate as well. The most striking characteristic of this dataset is the large-scale sigmoidal bedding with down lap, top lap, on the lap and truncation constructions. The biogenic gas pockets also generate bright spots, but are not widespread. It is possible to distinguish several seismic facies like a clear, chaotic, linear and shingle.

On the other hand, well logs indicate that transparent facies are of somewhat standardized lithology that can be either sand or shale. Probably slumping deposits are the irregular facies. It has been shown that shingles at the base of the clinoforms consist of sandy turbidity. The information was introduced with a median dip-steered filter with a two-trace radius to reduce the noise.

\section{Methodology}

In this study, two methods, namely the model-based seismic inversion (MBI) and MLFN were employed for data analysis. The MBI method is used to measure subsurface $P$-impedance (acoustic impedance). MLFN is implemented for porosity prediction in the inter-well region using inversion-derived acoustic impedance (AI) as input. The flowchart of the methodology is shown in Figure $2 a$, and MBI and MLFN techniques are described concisely in the subsequent subsections.

\section{Model-based inversion}

The seismic inversion is a subsurface modelling technique which generates geological structures utilizing seismic information as input and well logs as control. The MBI is 


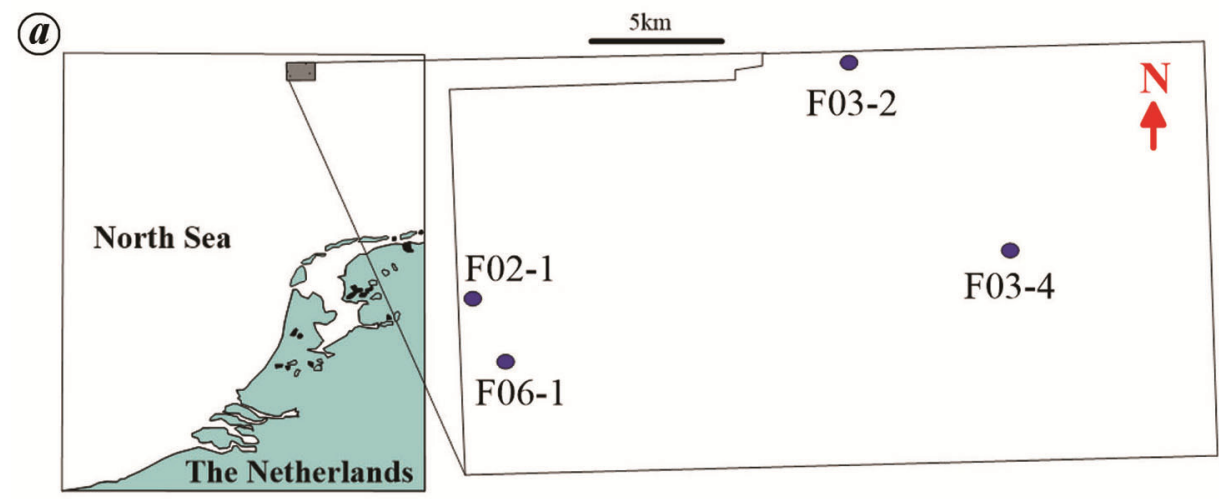

(b)

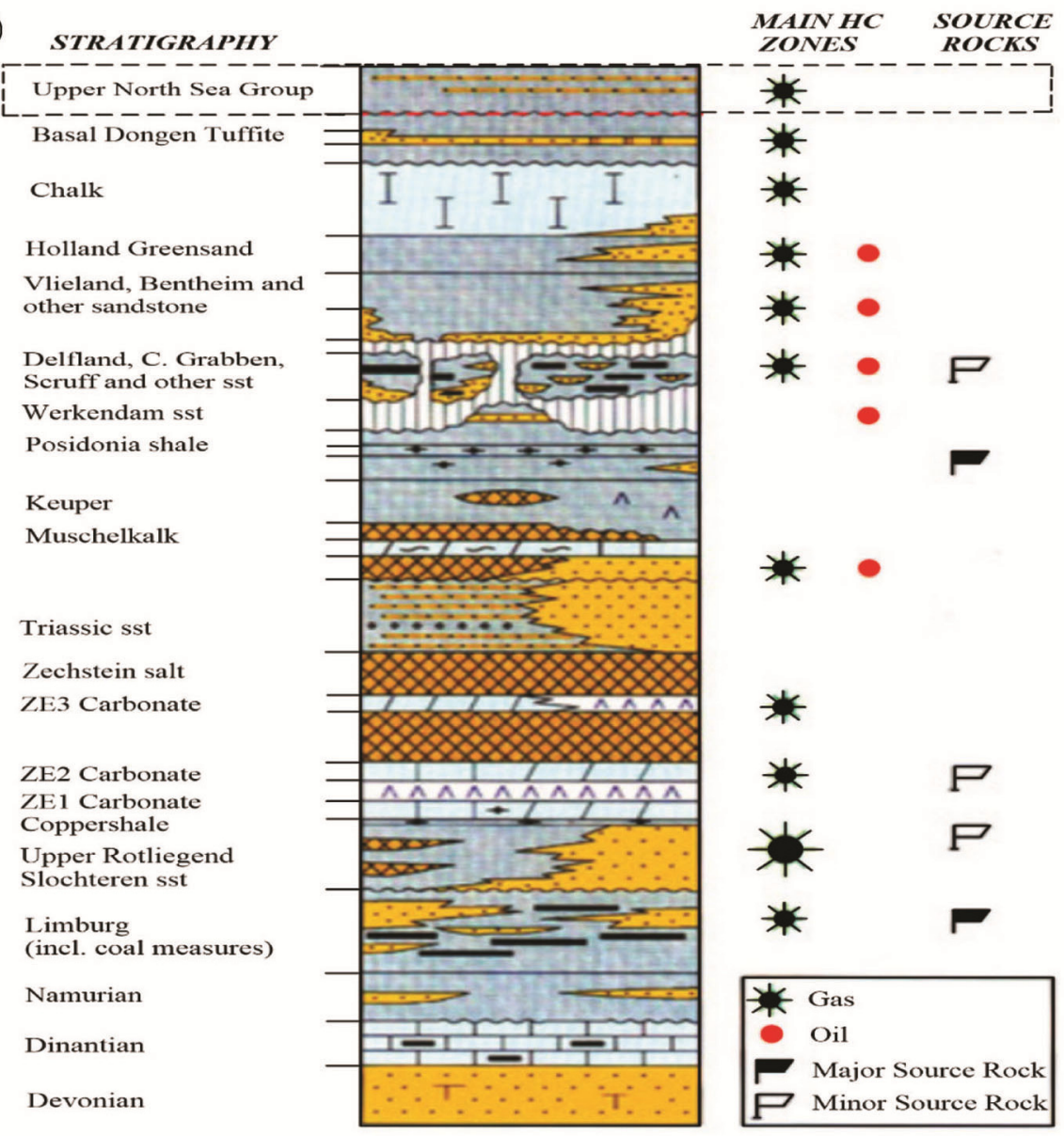

Figure 1. $\boldsymbol{a}$, Location map of the study area, F3 block, North Sea, the Netherlands. $\boldsymbol{b}$, Stratigraphy of the North Sea Region ${ }^{20}$.

a method of converting seismic amplitude to impedance value, which makes use of stacked (zero-offset) seismic information to provide depth or time pictures of AI. Also, $\mathrm{AI}$ is one of the parameters of rock physics that is determined by depth, porosity, fluid content, lithology type, pressure and temperature ${ }^{25-27}$

The fundamental of seismic exploration is to send a signal into the earth, which is recorded after reflection from a reflector. If the incidence angle is zero and the layers are flat, the Zoeppritz equations can be simplified as

$$
R_{i}=\frac{Z_{i+1}-Z_{i}}{Z_{i+1}+Z_{i}}
$$

where $R_{i}$ is the $P$-wave reflection coefficient (zero offset) at the $i$ th interface and $Z$ is the layer impedance, whereas $Z_{i}=\rho_{i} \times V_{i}$ for the $i$ th layer. 

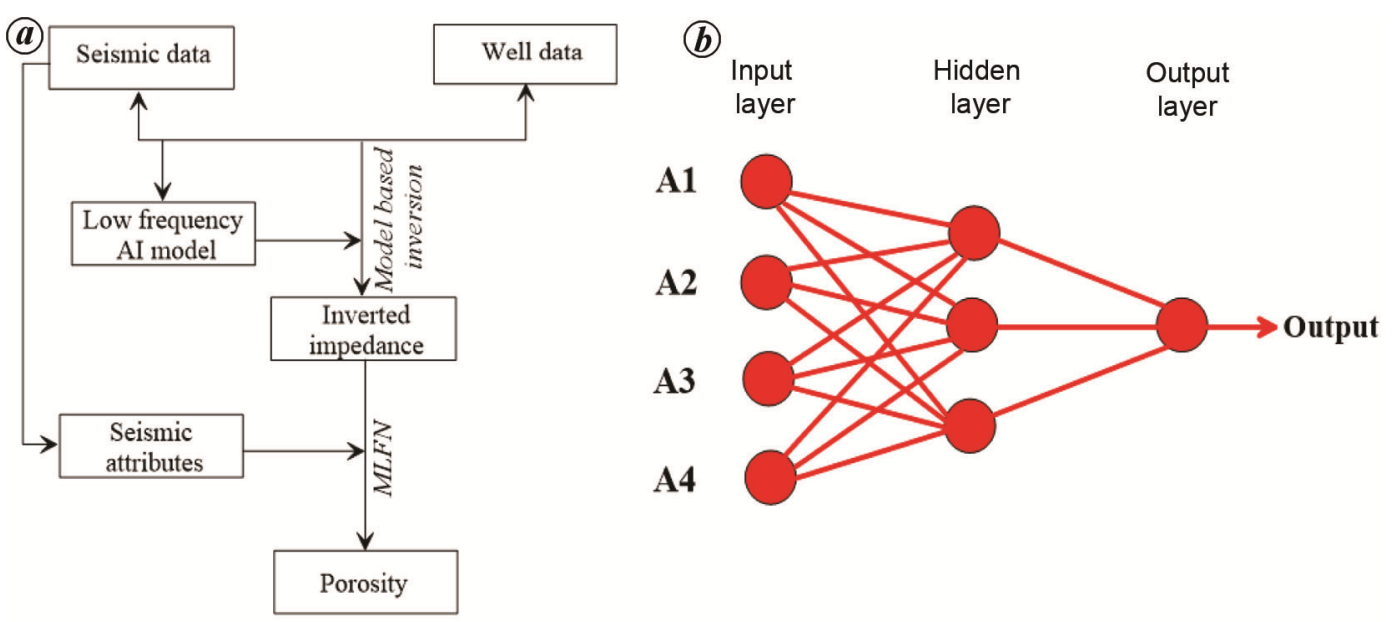

Figure 2. $\boldsymbol{a}$, Flowchart of the methodology implemented in the present study. $\boldsymbol{b}$, Architecture of multi-layer feedforward neural network (MLFN).

The reflection coefficient can be estimated from seismic reflection data using eq. (1). The seismic traces are produced by the convolution of a seismic wavelet with reflectivity with some additive noise. Mathematically, it can be represented as

$$
S(t)=[W(t) * R(t)]+N(t)
$$

where $S(t)$ represents seismic trace, $W(t)$ the seismic wavelet, $R(t)$ the reflectivity, * the convolution operator and $N(t)$ is the noise component of the data.

Lindseth $^{14}$ has demonstrated that if the recorded seismic signal is zero-offset signal, then it is possible to obtain the acoustic impedance in the next layer $Z_{i+1}$ as

$$
Z_{i+1}=Z_{i}\left[\frac{1+R_{i}}{1-R_{i}}\right] \text {. }
$$

Using eq. (3), the seismic reflection information can be iteratively transformed into $P$-impedance. For the first layer, acoustic impedance needs to be determined from an uninterrupted layer above the target region. In this method, impedance of the $n$th layer can be computed as

$$
Z_{n}=Z_{1} * \Pi\left[\frac{1+R_{i}}{1-R_{i}}\right]
$$

However, this method is not free from problems. The biggest drawback of this method is that the effect of the band-limited wavelet is expected to eliminate the lowfrequency reflectivity component and use the trace as a group of reflective coefficients, implying that it can never be accurately recovered following eq. (2). Another major problem of inversion is that it includes removing noise components and adequate seismic information scaling ${ }^{16}$. To solve these problems extra information is required, which is taken from the well logs. The logging information with stacked seismic information is added to ensure a more precise outcome from the seismic inversion. An inversion approach is one in which a primary lowfrequency model is iteratively improved to provide the best match for seismic data.

The problem encountered in recursive inversion is avoided in model-based inversion methods by iteratively reducing the error between modelled data and seismic data in the root mean square sense. The model-based inversion is the latest inversion method based on the model of convolution. If noise is uncorrelated to the seismic signal, one can describe the reflectivity following eq. (2). It is an equation that is nonlinear and band-limited, and can be solved iteratively. This alternative improves the fit between synthetic traces and the seismic information observed gradually.

\section{Multi-layer feed-forward neural network}

The neural network method in geophysics is used to predict subsurface petrophysical parameters away from the boreholes $^{9,28-32}$. These petrophysical parameters are beneficial in determining the underground reservoir and finding its horizontal extension. The neural network is divided into two categories; the first is MLFN, and the second is the probabilistic neural network (PNN). By considering geophysical data, these methods show different characteristics and hence cannot be used randomly. Liu and $\mathrm{Liu}^{33}$ defined the advantage of MLFN over PNN in the application of geophysical data. Therefore, in this study, MLFN is used to estimate subsurface porosity away from the borehole. Figure $2 b$ shows the basic architecture of MLFN. 
The MLFN network architecture is composed of one input layer, one or more hidden layers and one output layer. Each network layer comprises nodes that are connected and referred to as weights ${ }^{13}$. Further, these connections or weights are the final components of the output. The number of nodes depends on the number of attributes to be used in the MLFN process. Further, the number of desired attributes is decided by the convolution process. In the convolution process, each possible set of attributes is tested, and the set with maximum likeness is the desired one. Mathematically, the MLFN process is as follows.

The mapping function $\Gamma$ can be used for the formal description of the neurons, which assigns every neuron $i$ a subset $\Gamma(i) \subseteq V$, that consists of all the given ancestors of the neuron. A subset $\Gamma^{-1}(i) \subseteq V$ consists of all neuron $i$ predecessors. In the next layer, each neuron in a specific layer is linked to all neurons ${ }^{30}$. The connection between the $i$ th and $j$ th neurons is characterized by the $\omega_{i j}$ weight coefficient and the $v_{i}$ threshold coefficient of the $i$ th neuron. The weight coefficient represents the degree of significance in the neural network of the specified relationship $^{31}$. Equations (5) and (6) determine the $i$ th neuron output value (activity) of $x_{i}$.

$$
\begin{aligned}
& x_{i}=f\left(\psi_{i}\right), \\
& \psi_{i}=v_{i}+\sum_{j \in \Gamma_{i}^{-1}} \omega_{i j} x_{j},
\end{aligned}
$$

where $\psi_{i}$ represents the potential of the $i$ th neuron and $f\left(\psi_{i}\right)$ is the transfer function (the summation in eq. (6) is performed over all neurons $j$ carrying the signal to the $i$ th neuron). The transfer function is used for transformation purposes $^{31}$. In machine learning, the sums of each node are weighted, and the sum is passed through a nonlinear function known as a transfer function to obtain an output. The threshold coefficient of the connection to a formally added neuron $j$ can be assumed as a weight coefficient, where $x_{j}=1$. For the transfer function, it holds that

$$
f\left(\psi_{i}\right)=\frac{1}{1+\exp (-\psi)} .
$$

The monitored adaption method changes with the threshold coefficients $v_{i}$ and weight coefficients $\omega_{i j}$ to minimize the sum of the square errors between the calculated and necessary output values ${ }^{34}$. This is achieved by reducing the objective function $E$, which can be written as

$$
E=\sum_{p} \frac{1}{2}\left(x_{p}-\hat{x}_{p}\right)^{2},
$$

where $x_{p}$ and $\hat{x}_{p}$ are the vectors containing the measured and required activity of the output neurons and the summation runs across all output neurons $p$.

\section{Results and discussion}

This study describes the role of MBI and MLFN in the estimation of subsurface rock properties. The modelbased inversion is implemented to calculate the volume of $P$-impedance of the subsurface. The inversion is performed in two steps. First, a composite trace is extracted from the seismic data near the well location and inversion is performed, and output is compared with the original impedance from the well logs. Second, it is implemented to the entire seismic volume to convert the seismic amplitudes into AI.

Figure 3 represents log plots of gamma-ray, $P$-wave velocity, density, porosity and impedance. Figure $3 a$ presents plots of well-log properties for 1000-1500 m. Track 1 of Figure $3 a$ shows the variation of gamma-ray logs, track 2 shows velocity, track 3 shows the density plot, track 4 shows the porosity plot and track 5 shows impedance plots. Figure $3 b$ shows plots of well-log properties only for $1200-1300 \mathrm{~m}$. The analysis of $\log$ properties depicts a high gamma ray, low porosity and high velocity, high density and high impedance up to a depth of $1285 \mathrm{~m}$, whereas beneath this zone reverse characteristic can be noticed. The analysis also indicates that the area may have two anomalous zones near 1350 and $1450 \mathrm{~m}$. For further study, cross plots among velocity, density, impedance and porosity are plotted (Figure 4). Figure $4 a$ shows a cross plot between density and velocity, Figure $4 b$ shows a cross plot between velocity and porosity, and Figure $4 c$ shows a cross plot between porosity and AI. These cross plots also clearly suggest the presence of anomalous zones.

Figure 5 presents the inversion results from 800 to $1625 \mathrm{~ms}$. Track 1 shows inversion results, track 2 shows synthetic trace, track 3 shows seismic trace and track 4 shows error trace. In track 1 , the solid red line indicates inverted impedance, the solid blue line shows original impedance from well $\operatorname{logs}$ and the solid black line depicts the initial model of impedance. From Figure 5, it can be noticed that the inverted impedance follows well the trend of the original impedance. The results are presented for well F02-1; the results are also generated for well F06-1. Table 1 shows the statistical comparisons for both the wells. The statistical analysis demonstrates that the performance of MBT is excellent. Further, a cross plot of original and inverted impedance is estimated to crossverify inversion results (Figure 6). The distribution of points is near the best-fitted line, which indicates that the original and inverted impedances are close enough to each other.

Thereafter, inversion of the entire seismic volume was performed, and impedance volume estimated. Figure 7 shows the cross-section of inverted impedance. Figure $7 a$ shows a cross-section at inline 244 and Figure $7 b$ shows a cross-section at inline 362. Acoustic impedance from well-log data is also plotted along a column on the 

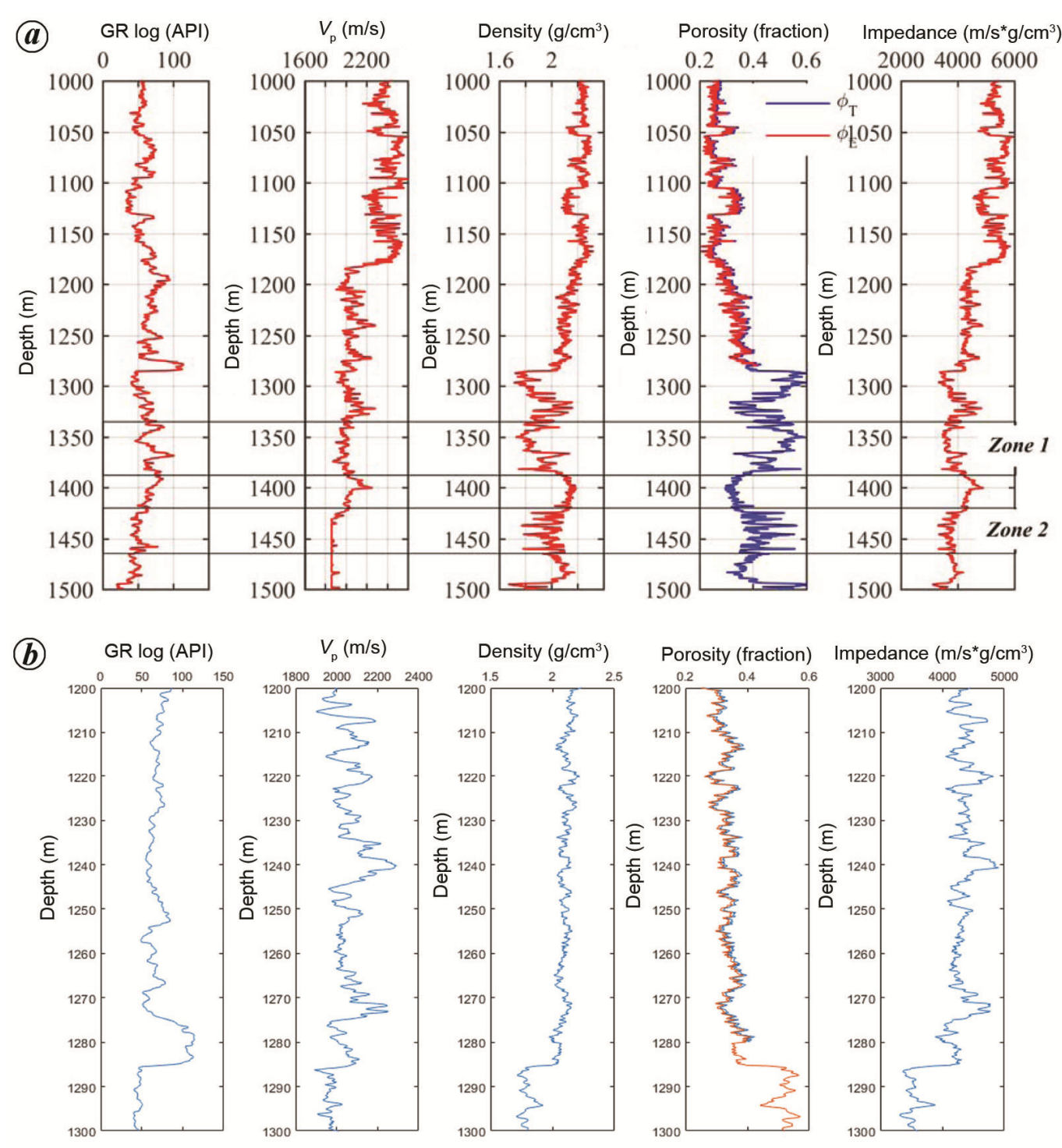

Figure 3. $\boldsymbol{a}$, Variation of well-log curves with depth. Two anomalous zones are highlighted by the rectangle. $\boldsymbol{b}$, Plots of well-log properties only for $1200-1300 \mathrm{~m}$.
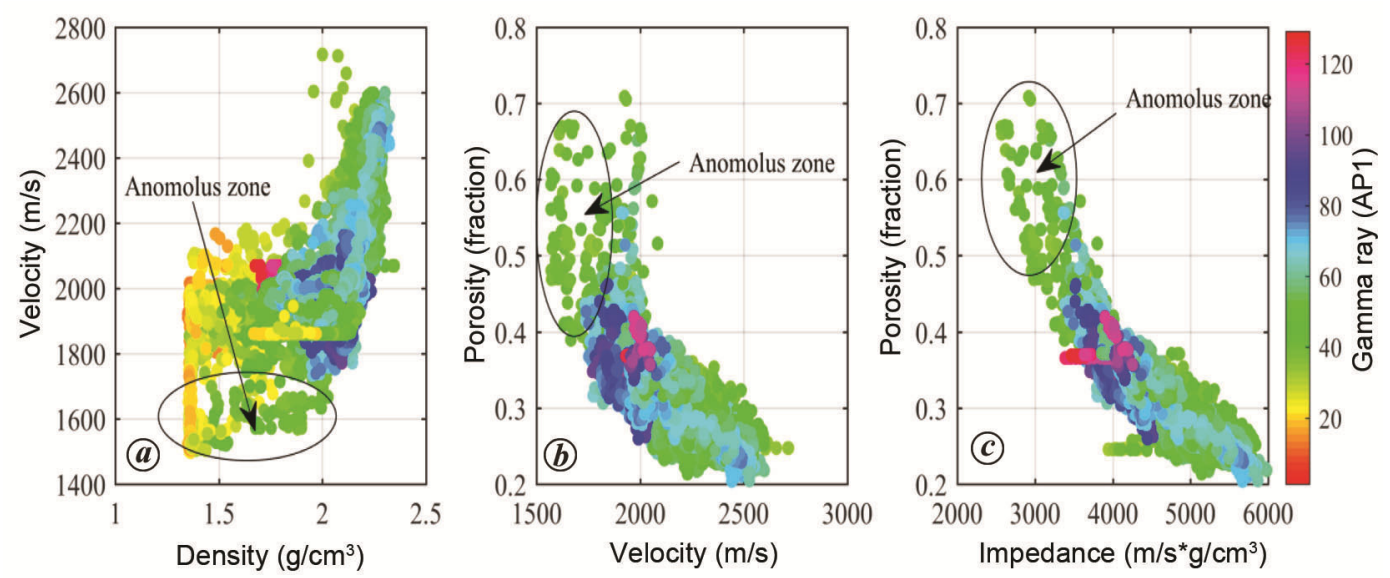

Figure 4. Cross plot between $(\boldsymbol{a})$ density and velocity, $(\boldsymbol{b})$ velocity and porosity, and $(\boldsymbol{c})$ impedance and porosity. The colour bar shows gamma-ray variation. The ellipse highlights the anomalous zone in the figure. 


\section{RESEARCH ARTICLES}

inverted section. It can be noticed that the inverted impedance matches well with the well-log impedances. As the porosity prediction using MLFN is mainly dependent on these inverted sections, hence at every step quality check (QC) of the inverted results is performed. The inverted impedance shows a variation of 2500 to $6200 \mathrm{~m} / \mathrm{s}^{*} \mathrm{~g} / \mathrm{cm}^{3}$, which is quite low. From this low impedance, one can infer the presence of loose soil formation. From the inverted section, it may be concluded that there is no primary anomalous zone in the area.

Table 1. Statistical comparison of seismic inversion results

\begin{tabular}{lccc}
\hline Well & Correlation & Synthetic error & $\begin{array}{c}P \text { impedance error } \\
\left(\mathrm{m} / \mathrm{s}^{*} \mathrm{~g} / \mathrm{cm}^{3}\right)\end{array}$ \\
\hline F02-1 & 0.97 & 0.246 & 418.8 \\
F06-1 & 0.99 & 0.138 & 537.5 \\
\hline
\end{tabular}

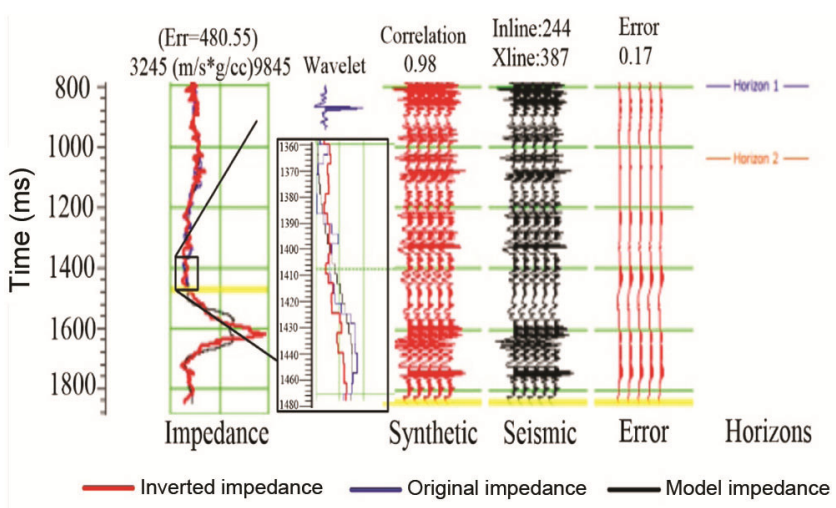

Figure 5. Inversion analysis results. The first track compares inverted acoustic impedance AI (red line) with original AI (blue line) along with the initial AI model (black line). The second track shows a correlation between original and inverted curves. The track third shows a seismic trace close to well location F02-1, and the fourth track shows error variation.

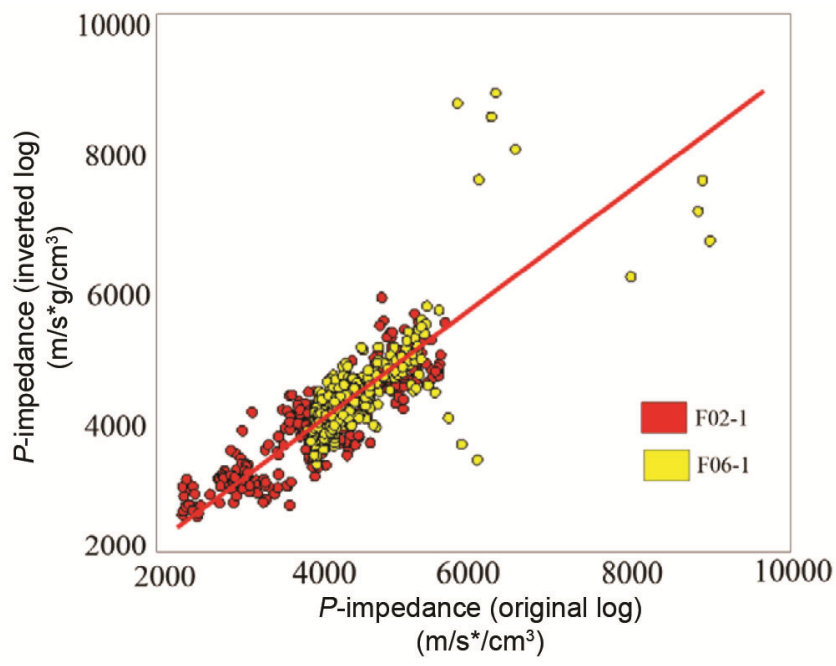

Figure 6. Cross plot between original impedance and inverted impedance for both wells F02-1 and F06-1. The solid red line represents the best-fit line of data.
Next, the MLFN tool was applied to the data and porosity volume estimated. MLFN analyses several attributes to be utilized in the process. Table 2 lists the analysed attributes. The first column of Table 2 shows the target log, the second column shows attribute names, the third shows training error and the fourth column shows the validation error. From this table, it can be seen that the training error decreases for the first two attributes, and thereafter it starts increasing for more than two attributes. Hence the first two attributes, namely $\log \left(\right.$ inverted $\left.Z_{p}\right)$ and Quadrature trace were used to predict the porosity.

The prediction was implemented in two steps. Firstly, it was applied to the composite seismic trace close to wells to verify prediction results. Secondly, it was adapted to the entire seismic volume to predict porosity volume. Figure 8 compares the predicted porosity with the original porosity. Figure $8 a$ compares porosities for well F021 , whereas Figure $8 b$ shows the difference between these two curves. Figure $8 c$ shows the comparison for well F06-1, whereas Figure $8 d$ shows the difference between predicted and original curves. The actual and predicted porosities matched well with each other for both wells. The correlation coefficient between the original and predicted porosity was 0.90 , and RMS error between them was 0.028 , which is within a reasonable range and indicates good prediction results.

Figure 9 presents the cross plot between the predicted and actual porosities (for wells F02-1 and F06-1). From the figure, we can observe that the plotted points fall close to the best-fitted lines, which again reveals good prediction results.

The MLFN tool was applied to the entire seismic volume to predict porosity. Figure 10 presents the porosity cross-section. Figure $10 a$ shows the porosity crosssection at inline 244, whereas Figure $10 \mathrm{~b}$ shows the same for inline 362. These sections show that the study area has a porosity ranging from 0.07 to 0.40 . The estimated porosity section depicts very high-resolution images of the subsurface compared to the seismic section. The welllog porosity curves were also plotted over the predicted porosity volume to observe the matching. Figure 10 shows that the predicted porosity section has a perfect match with the well-log porosity curve. Analysis of the inverted section indicates the presence of no primary anomaly zone and hence no major reservoir in the area. This conclusion is valid for a small section of the area, which has been analysed in this study. However, the other parts of the region may have different characteristics.

Figure 11 shows a cross plot between impedance and porosity for well F02-1. This cross plot was generated to measure the mathematical relationship between porosity and impedance of the area. The estimated relationship is

$$
\phi=3.8 \times 10^{-8} \mathrm{AI}^{2}-0.00044 \mathrm{AI}+1.5
$$


RESEARCH ARTICLES

Table 2. Multiple attribute correlation results: multi attribute list 1

\begin{tabular}{llcc}
\hline Target & \multicolumn{1}{c}{ Final attribute } & $\begin{array}{c}\text { Training error } \\
\text { (fraction) }\end{array}$ & $\begin{array}{c}\text { Validation error } \\
\text { (fraction) }\end{array}$ \\
\hline Sqrt (porosity) & Log (inverted_Zp) & 0.028014 & 0.030149 \\
Sqrt (porosity) & Quadrature trace & 0.024582 & 0.026881 \\
Sqrt (porosity) & Filter 15/20-25/30 & 0.024210 & 0.027900 \\
Sqrt (porosity) & Cosine instantaneous phase & 0.023959 & 0.027736 \\
Sqrt (porosity) & Amplitude weighted frequency & 0.023700 & 0.027499 \\
Sqrt (porosity) & Apparent polarity & 0.023458 & 0.027609 \\
Sqrt (porosity) & Filter 35/40-45/50 & 0.023199 & 0.027596 \\
Sqrt (porosity) & Integrated absolute amplitude & 0.023094 & 0.030859 \\
\hline
\end{tabular}

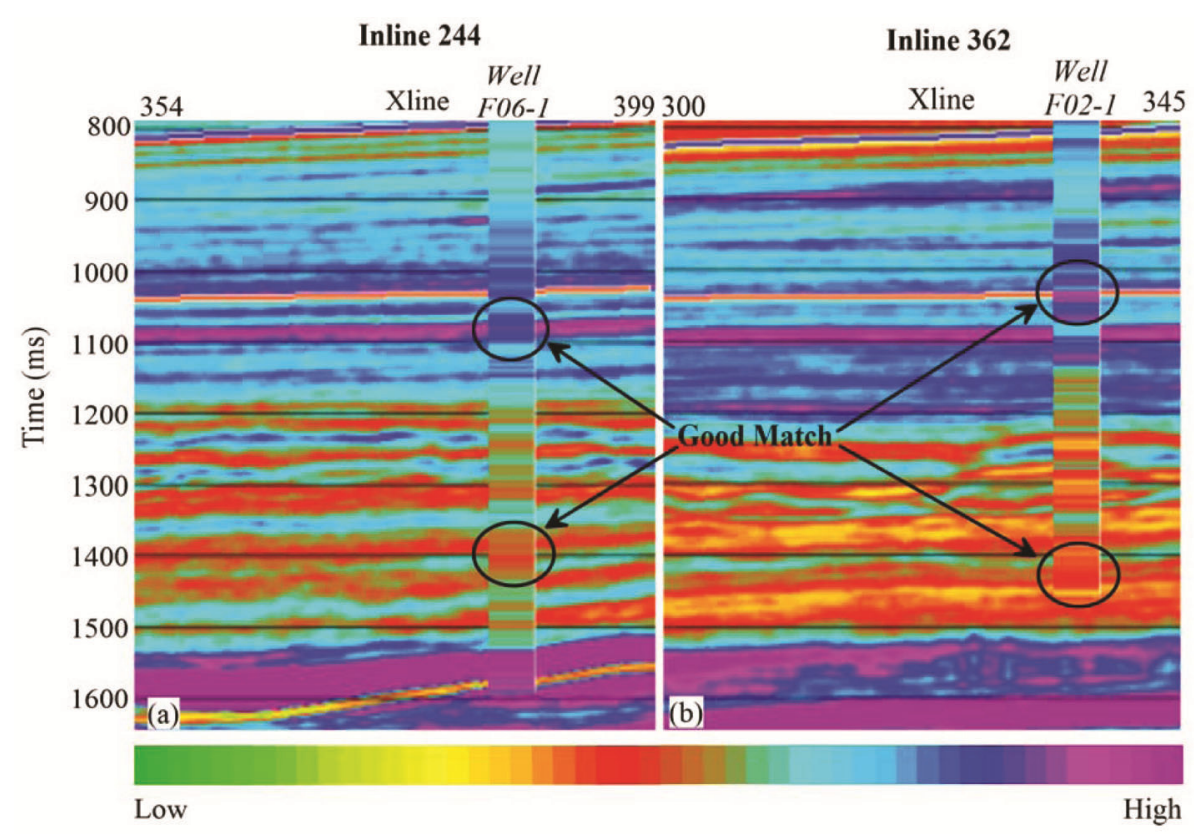

Figure 7. Cross-sections of the inverted impedances at (a) inline 244 and (b) inline 362 . The well-log impedance from wells F02-1 and F06-1 is also plotted on the inverted sections along the column.

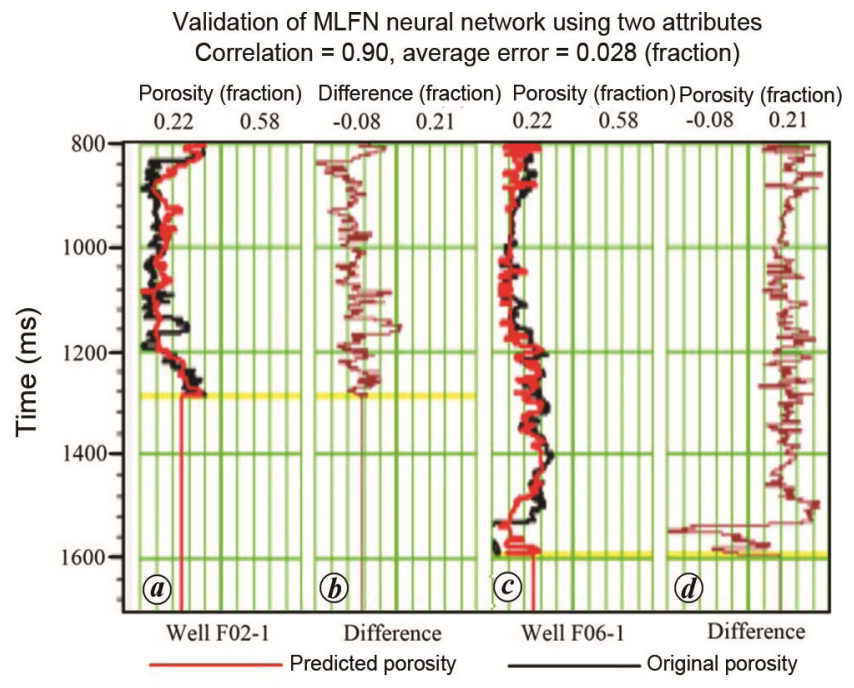

Figure 8. Track 1 shows curves of predicted porosity and original porosity from the well F02-1 and track 2 shows the difference between them. Track 3 shows a comparison of predicted porosity with the original porosity from well F06-1 and track 4 shows the difference between them.

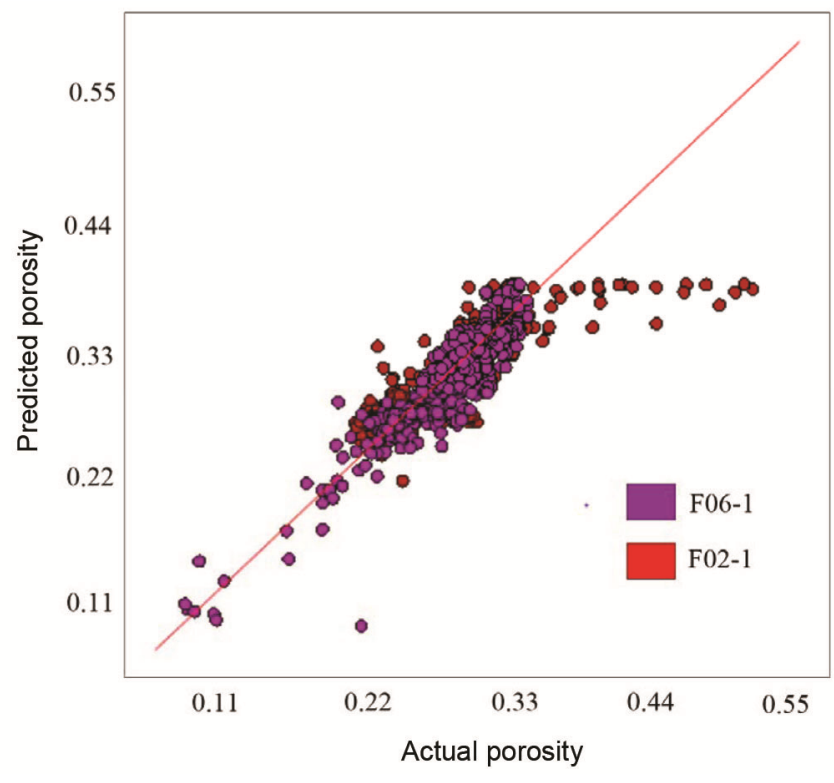

Figure 9. Cross plot between actual porosity and predicted porosity for both wells (F02-1 and F06-1). 


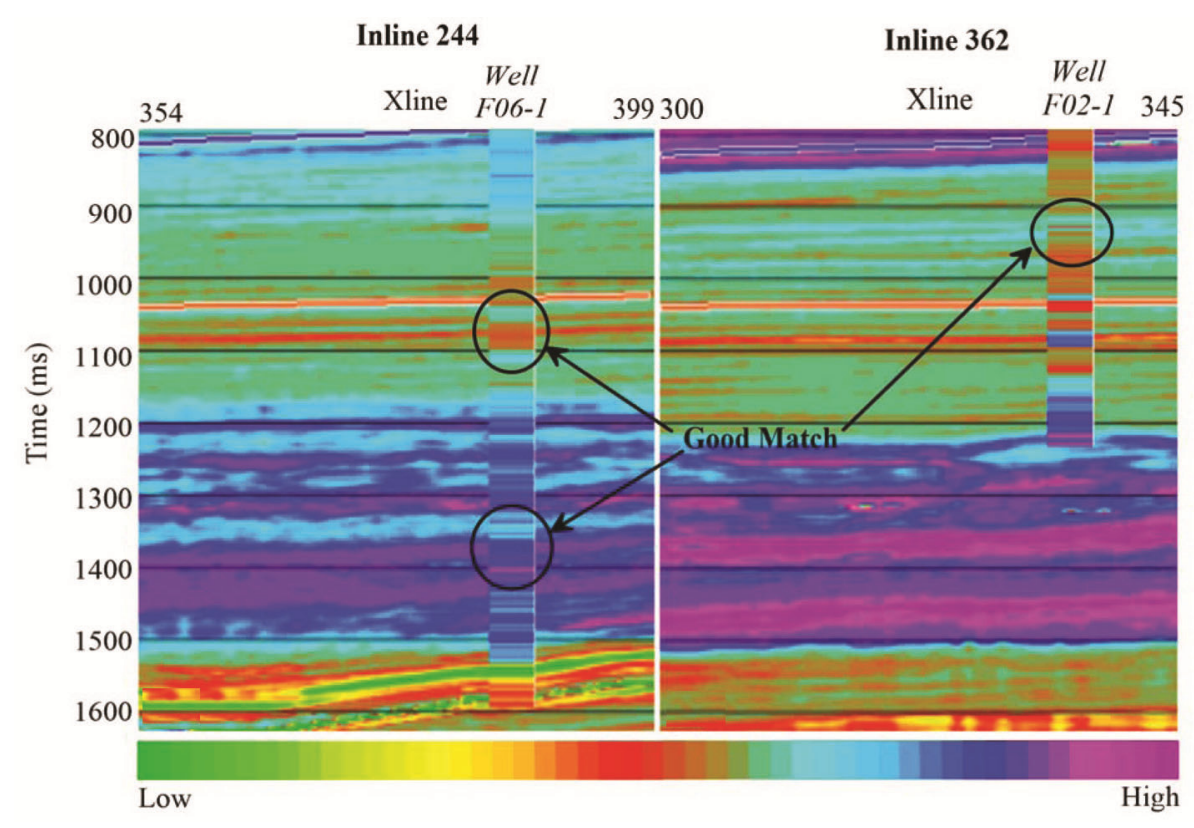

Figure 10. Cross-sections of predicted porosities at $(\boldsymbol{a})$ inline 244 and $(\boldsymbol{b})$ inline 362 . The well-log porosities (F02-1 and F06-1) are also shown along the column on the predicted sections.

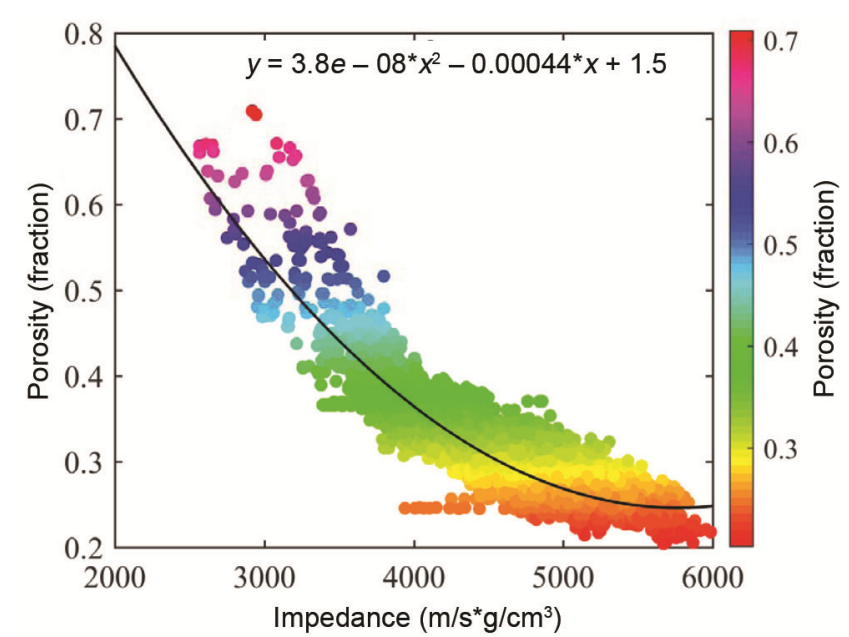

Figure 11. Cross plot between original impedance and porosity from well F02-1. The best-fit line gives a relation between them.

The cross plot between inverted impedance and predicted porosity (Figure 12), provides a relationship between porosity and impedance as

$$
\phi=-2.4 \times 10^{-8} \mathrm{AI}^{2}+0.00017 \mathrm{AI}+0.061 \text {. }
$$

These relationships are significant as one can estimate the porosity section from the impedance section without performing MLFN inversion. Equations (9) and (10) both give the relationship between impedance and porosity, but eq. (9) is generated using information from a particular location (well F02-1), whereas eq. (10) is generated using information from the region (seismic section).
Figure 13 shows the predicted porosity in well F06-1 using both the relationships. This has all three curves, original porosity from well F06-1 and predicted porosity from both the equations. From Figure 13, it can be observed that the predicted porosity from eq. (10) is closer to the original porosity from the well compared to predicted porosity computed using eq. (9). The correlation between original porosity and predicted porosity calculated using eq. (9) is estimated to be 0.83 , whereas the correlation between actual porosity and predicted porosity computed using eq. (10) is found to be 0.89 . This analysis proves that the relationship derived from the predicted and inverted section is valid for the entire region, whereas the relationship derived from well $\operatorname{logs}$ is valid to only near the well locations. Hence one can use equations derived from predicted porosity and inverted impedance to estimate the porosity of a region without using the MLFN method.

\section{Conclusion}

In the present study, vital rock parameters, namely impedance and porosity, were estimated from the inversion of offshore seismic data of F3 Block, the Netherlands. The MLFN algorithm was used to predict porosity away from the boreholes. For this purpose, initially MBI was used to estimate subsurface AI. Then this impedance, along with other attributes, was used to predict porosity in the inter-well region using MLFN as a prediction tool. The study aims to establish a relationship between porosity and impedance, and find the prospective zone, if any. Initially, the MBI method is applied to the composite 
seismic trace close to the well location and inverted for the impedance curve. Thereafter, the entire seismic section volume was inverted for the impedance volume. The area has impedance varying from 2500 to $6200 \mathrm{~m} / \mathrm{s}^{*} \mathrm{~g}$ / $\mathrm{cm}^{3}$, a relatively low value, which indicates that the area has less compact rocks. It is also noticed that there is no

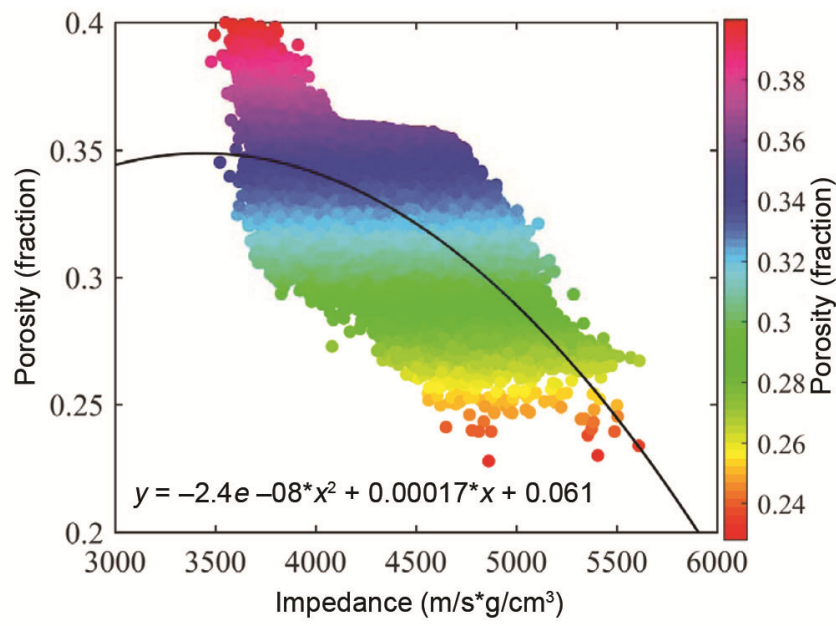

Figure 12. Cross plot between inverted acoustic impedance and predicted porosity volume. The best-fit line provides a relationship between porosity and impedance of the region.

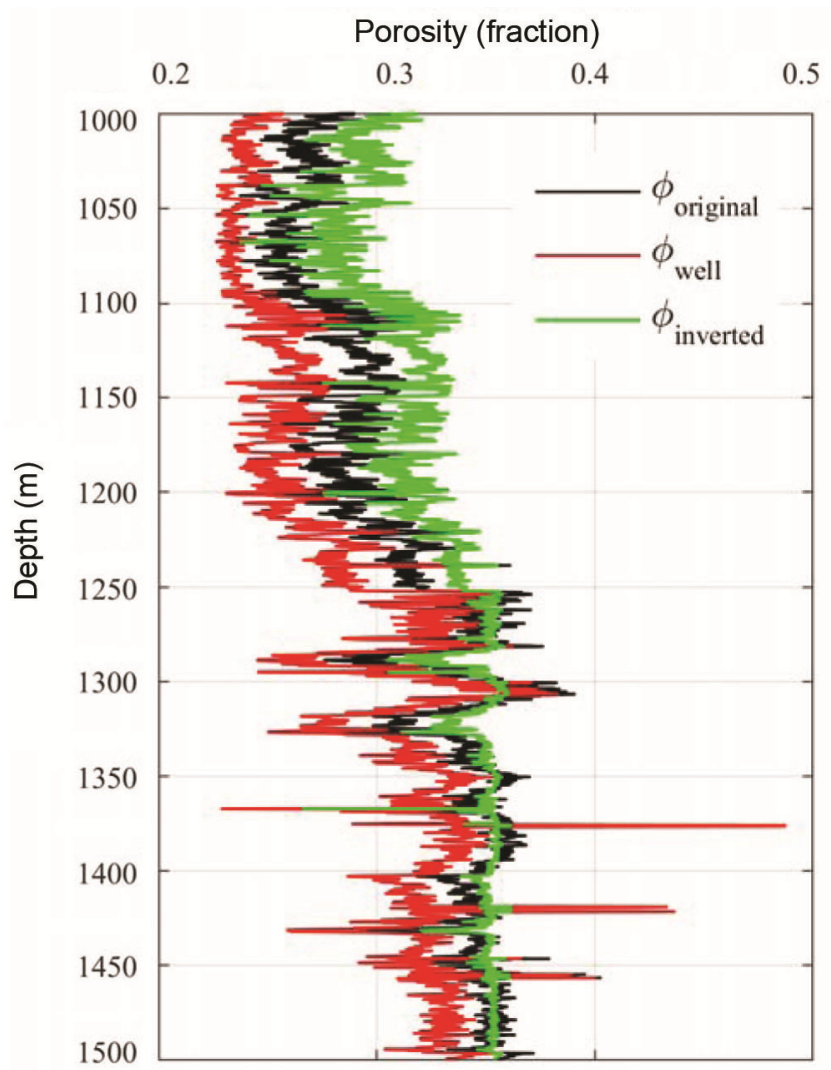

Figure 13. Plots of predicted porosity using eqs (9) and (10) along with well-log porosity from well F06-1. significant anomalous zone in the area. Next, porosity in the inter-well region was predicted using MLFN. The variation of porosity was found to be $0.07-0.40$, which is comparatively high and thereby confirms that the area has less compact formation. The correlation of predicted porosity with actual porosity was found to be 0.89 , a high value which indicates excellent performance of MLFN for offshore data of F3 block, the Netherlands. The section also suggests that there is no primary anomalous zone and hence non-existence of a reservoir. Further, the inverted and predicted sections were used to estimate the relationship between porosity and impedance. It was observed that the derived relationship showed better prediction than the one derived from well-log data.

1. Oldenburg, D. W., Scheuer, T. and Levy, S., Recovery of the acoustic impedance from reflection seismograms. Geophysics, 1983, 48, 1318-1337.

2. Kushwaha, P. K., Maurya, S. P., Singh, N. P. and Rai, P., Estimating subsurface petro-physical properties from raw and conditioned seismic reflection data: a comparative study. J. Indian Geophys. Union, 2019, 23, 285-306.

3. Kumar, D., Sugianto, H., Li, S., Patel, H. and Land, S., Using relative seismic impedance to predict porosity in the Eagle Ford shale. In SEG Technical Program Expanded Abstracts 2014, Society of Exploration Geophysicists, Keystone, Colorado, USA, 2014, pp. 2688-2692.

4. Yilmaz, O., Seismic data analysis: processing, inversion, and interpretation of seismic data. Society of Exploration Geophysics, Tulsa, 2001.

5. Dangwal, N. S., Mohanty, S. K., Manna, D., Singh, B. and Verma, N. K., Refinement of sand dispersal pattern in deltaic environment using multimineral $\log$ processing and multiattribute analysis - a case study in Nardipur Low, Cambay Basin, India. In 0Geo India Conference and Exhibition Greater Noida, New Delhi, 2008.

6. Doyen, P. M., Porosity from seismic data: a geostatistical approach. Geophysics, 1988, 53, 1263-1275.

7. Maurya, S. P. and Singh, N. P., Seismic modelling of $\mathrm{CO}_{2}$ fluid substitution in a sandstone reservoir: a case study from Alberta, Canada. J. Earth Syst. Sci., 2019, 128, 236.

8. Haas, A. and Dubrule, O., Geostatistical inversion - a sequential method of stochastic reservoir modelling constrained by seismic data. First Break, 1994, 12, 561-569; https://doi.org/10.3997/ 1365-2397.1994034.

9. Russell, B., Hampson, D., Schuelke, J. and Quirein, J., Multiattribute seismic analysis. Lead. Edge, 1997, 16, 1439-1444.

10. Hampson, D. P., Schuelke, J. S. and Quirein, J. A., Use of multiattribute transforms to predict $\log$ properties from seismic data. Geophysics, 2001, 66, 220-236.

11. Kushwaha, P. K., Maurya, S. P., Singh, N. P. and Rai, P., Use of maximum likelihood sparse spike inversion and probabilistic neural network for reservoir characterization: a study from F-3 block, the Netherlands. J. Pet. Explor. Prod. Technol., 2020, 10, $829-845$.

12. Bosch, M., Mukerji, T. and Gonzalez, E. F., Seismic inversion for reservoir properties combining statistical rock physics and geostatistics: a review. Geophysics, 2010, 75, 75A165-75A176; https://doi.org/10.1190/1.3478209.

13. Maurya, S. P., Singh, N. P. and Singh, K. H., Seismic Inversion Methods: A Practical Approach, Springer Science and Business Media LLC, Springer Nature Switzerland AG, 2020.

14. Lindseth, R. O., Synthetic sonic logs - a process for stratigraphic interpretation. Geophysics, 1979, 44, 3-26. 
15. Aminzadeh, F. and De Groot, P., Neural Networks and Other Soft Computing Techniques with Applications in the Oil Industry. EAGE Book Series, Houten, The Netherlands, 2006.

16. Maurya, S. P., Singh, K. H. and Singh, N. P., Qualitative and quantitative comparison of geostatistical techniques of porosity prediction from the seismic and logging data: a case study from the Blackfoot Field, Alberta, Canada. Mar. Geophys. Res., 2019, 40, 51-71.

17. Chambers, R. L., Zinger, M. L. and Kelly, M. C., Constraining geostatistical reservoir descriptions with 3-D seismic data to reduce uncertainty. In Stochastic Modeling and Geostatistics: Principles, Methods, and Case Studies (eds Yarus, J. and Chambers, R.), AAPG Computer Applications in Geology 3, 1994, pp. $143-157$.

18. De Jager, J., Geological development. In Geology of the Netherlands (eds Wong, T. E., Batjes, D. A. J. and De Jager, J.), Royal Netherlands Academy of Arts and Sciences, Amsterdam, 2007.

19. Michalowicz, P., Three dimensional model of the geological structure of the F3 block. Researchgate.net publication. id 308597487, 2016.

20. Rondeel, H. E., Batjes, D. A. J. and Nieuwenhuijs, W. H. (eds), Geology of Gas and Oil under the Netherlands, Selection of papers presented at the 1993 International Conference of the American Association of Petroleum Geologists, Springer Science and Business Media, 1996.

21. De Bruin, G. and Bouanga, E. C., Time attributes of stratigraphic surfaces, analysed in the structural and wheeler transformed domain. In 69th EAGE Conference and Exhibition incorporating SPE EUROPEC 2007. European Association of Geoscientists and Engineers, London, UK, 2007.

22. Wolak, J., Hemstra, N., Ochoa, J. and Pelissier, M., Reconstruction of depocenter evolution through time using relative stratigraphic thickness. Lead. Edge, 2013, 32, 172-177.

23. Overeem, I., Weltje, G. J., Bishop-Kay, C. and Kroonenberg, S B., The Late Cenozoic Eridanos delta system in the southern North Sea Basin: a climate signal in sediment supply? Basin Res., 2001, 13, 293-312.

24. Sørensen, J. C., Gregersen, U., Breiner, M. and Michelsen, O., High-frequency sequence stratigraphy of Upper Cenozoic deposits in the central and southeastern North Sea areas. Mar. Pet. Geol., 1997, 14, 99-123.

25. Maurya, S. P. and Singh, K. H., Band limited impedance inversion of Blackfoot field, Alberta, Canada. J. Geophys., 2017, 38, 57-61.
26. Kushwaha, P. K., Maurya, S. P., Singh, N. P. and Rai, P., Use of maximum likelihood sparse spike inversion for reservoir characterization - a case study from F-3 Block, Netherland. J. Geophys., 2019, 43, 127-133.

27. Kushwaha, P. K., Richa, Maurya, S. P., Rai, P. and Singh, N. P., Comparison of band-limited and coloured seismic inversion methods to estimate acoustic impedance of F3 block Netherlands a case study. In 13th Biennial International Conference and Exhibition, Society of Petroleum Geophysicist, Kochi, 2020.

28. Masters, T., Signal and Image Processing with Neural Networks, John Wiley and Sons, Inc., New York, USA, 1994.

29. Maurya, S. P. and Singh, K. H., Reservoir characterization using model-based inversion and probabilistic neural network. Discovery, 2015, 49, 122-127.

30. Maurya, S. P. and Singh, N. P., Application of LP and ML sparse spike inversion with probabilistic neural network to classify reservoir facies distribution - a case study from the Blackfoot field, Canada. J. Appl. Geophys., 2018, 159, 511-521.

31. McCormack, M. D., Neural computing in geophysics. Lead. Edge, 1991, 10, 11-15.

32. Schuelke, J. S., Quirein, J. A., Sarg, J. F., Altany, D. A. and Hunt, P. E., Reservoir architecture and porosity distribution, Pegasus field, West Texas - an integrated sequence stratigraphy-seismic attribute study using neural networks: SEG Technical Program, Expanded Abstracts, 1997, pp. 668-671.

33. Liu, Z. and Liu, J., Seismic-controlled non-linear extrapolation of well parameters using neural networks. Geophysics, 1998, 63, 2035-2041.

34. Pramanik, A. G., Singh, V., Vig, R., Srivastava, A. K. and Tiwary, D. N., Estimation of effective porosity using geostatistics and multiattribute transforms: a case study. Geophysics, 2004, 69, 352-372.

ACKNOWLEDGEMENTS. We thank CGG Veritas, a fully integrated geoscience company, for providing the Hampson Russell Suite, and the OpendTect Seismic Repository (dGB Earth Sciences) for providing open access seismic and well $\log$ data. We also thank University Grant Commission, Government of India, for a research grant to P.K.K. (CSIR-UGC NET-JRF/SRF Fellowship).

Received 30 March 2020; revised accepted 27 August 2020

doi: $10.18520 / \mathrm{cs} / \mathrm{v} 119 / \mathrm{i} 10 / 1652-1662$ 